\title{
The journal relative impact: an indicator for journal assessment
}

\author{
Elizabeth S. Vieira • José A. N. F. Gomes
}

Received: 30 March 2011 / Published online: 5 August 2011

(C) Akadémiai Kiadó, Budapest, Hungary 2011

\begin{abstract}
This paper presents the journal relative impact (JRI), an indicator for scientific evaluation of journals. The $J R I$ considers in its calculation the different culture of citations presented by the Web of Science subject categories. The JRI is calculated considering a variable citation window. This citation window is defined taking into account the time required by each subject category for the maturation of citations. The type of document considered in each subject category depends on its outputs in relation to the citations. The scientific performance of each journal in relation to each subject category that it belongs to is considered allowing the comparison of the scientific performance of journals from different fields. The results obtained show that the JRI can be used for the assessment of the scientific performance of a given journal and that the SJR and SNIP should be used to complement the information provided by the JRI. The JRI presents good features as stability over time and predictability.
\end{abstract}

Keywords Normalization · Journal impact · Assessment $\cdot$ Variable window

\section{Introduction}

Journal evaluation is relevant as librarians, scientists, science evaluators, governments, policymakers and publishers need some sort of instrument to compare the value of journals. Librarians use journal evaluation to take decisions about subscriptions. Scientists want to choose the best and more appropriate journal to publish their research outputs. Governments want their researchers to publish in the most prestigious journals and science evaluators use the journal impact and prestige as elements for assessing the visibility of

E. S. Vieira · J. A. N. F. Gomes ( $\square)$

REQUIMTE/Departamento de Química e Bioquímica, Faculdade de Ciências, Universidade do Porto, Rua do Campo Alegre, 687, 4169-007 Porto, Portugal

e-mail: jfgomes@fc.up.pt

E. S. Vieira

REQUIMTE/Departamento Engenharia Industrial e Gestão, Faculdade de Engenharia, Universidade do Porto, Rua Dr. Roberto Frias, s/n, 4200-465 Porto, Portugal 
research activities. Publishers can associate high citation frequency to a successful editorial policy and use these data to advertise their journals in the scientific community. Citation counts have been used to assess the scientific performance of the documents published in a given journal since authors use citations to identify which publications contributed to the development of their research. This lead to the development of a set of impact indicators for journals that are normally based on citation counts. However, several authors also developed impact measures based on social network analysis (Leydesdorff 2007) and usage data (related with the number of downloads of papers from electronic publication archives) (Bollen and van de Sompel 2008; Shepherd 2010). These authors consider that by applying usage data the scientific activities can be evaluated immediately while the use of citations count introduces time lag of several years. Indicators based on citations counts are calculated using information from multidisciplinary databases such as ISI Web of Knowledge and Scopus. These databases encompass mainly peer-reviewed journals and thus citations are especially related with journal articles (Jacso 2005). In some fields as social sciences and humanities, researchers cite much more books than for example in chemistry or physics (Nederhof 2005) and therefore the use of impact indicators based on citation counts given by these databases has to be very careful. Several indicators for journal assessment have been developed in the last few years (Garfield and Sher 1963; Moed et al. 1998; Van Leeuwen and Moed 2002; Sombatsompop et al. 2005; Bollen et al. 2006; Zitt and Small 2008; Gonzalez-Pereira et al. 2010; Moed 2010). Some of these indicators consider in the normalization process the cited-side using the categorization of the scientific journals in the Web of Science (WoS) subject categories (Moed et al. 1998; Van Leeuwen and Moed 2002). This type of normalization has been used since the 1970s (Murugesan and Moravcsik 1978). However, there are several criticisms to this type of normalization, especially, to the classification scheme used in the WoS (Opthof and Leydesdorff 2010). Other indicators use a process of normalization based on the citing-side (Zitt and Small 2008; Moed 2010) paying attention to all citations appearing in the current year of the documents published in the relevant journals in the past years. This type of normalization is not dependent on the classification of journals into fields. Citing-side normalization started being used in the 1980s (Small and Sweeney 1985). A totally different approach is the attempt to measure the influence of a journal as tried already in the 1970s (Pinski and Narin 1976), giving to each citation a weight dependent to the impact of the citing journal. Although initially proposed a long time ago, only recently have indicators based on the prestige of the citing journal been developed (Bergstrom 2007; Gonzalez-Pereira et al. 2010).

The different cultures of publication and citation of each scientific area play an important role. These differences are related with:

- Publication culture. Researchers in different fields tend, in average, to publish a widely varying number of documents and give, in each document, a different number of references. It is well know that for example in mathematics researchers publish and cite less than in fields as chemistry or biology (Vieira and Gomes 2010).

- Channel for dissemination of the results of the research activities. In social sciences and humanities researchers publish more in books than in journals or serials (Kyvik 2003); in computer science researchers publish their results more in conference proceedings than in journal articles (Moed and Visser 2007) and proceedings papers receive more citations than in other fields (Lisee et al. 2008).

- Time required for the maturation of the citations. The length of the citation windows used on the development of impact indicators should be selected carefully. In 
mathematics and engineering, citations mature more slowly than in fields related with the health sciences (Moed et al. 1998). This ageing process has been studied. Two methods have been discussed, the diachronous and the synchronous methods. In the diachronous methods citations of published documents in a given year are counted along several years. In the synchronous methods the citing year is fixed (one citing year normally). Glanzel and Schoepflin (1995) showed that the ageing process is characterized by two phases, maturation and decline and they identified several styles of ageing processes for different journals. Moed et al. 1998), analysed differences in journal rankings using normalized impact factors with short and long citations windows, considering different ageing processes. The authors observed, to a sample of journals indexed in the Journal Citation Reports (JCR), that a significant number of journals obtain the maximum impact only several years after publication (slowly maturation). They concluded that the Impact Factor available at the JCR is unfair to journals with slow maturation when compared with those journals with rapid maturation. This study showed the significance of the maturation of citations on the design of performance indicators.

- Multidisciplinarity effect. When a journal publishes work from several subject categories, its performance may be better when seen from the standpoint of one subject category than from the other. An indicator that considers the different performances of the journals in the fields they belong to should be considered.

Van Leeuwen and Moed (2002) presented the journal to field impact score (JFIS). This indicator is based on four types of documents and is a weighted field normalized indicator. The JFIS considers a flexible and variable citation and publication windows. The authors state that the length of the publication and citation windows may be longer than 5 years for humanities or mathematics.

The source normalized impact per paper (SNIP) (Moed 2010) does not require the classification of the journals into fields due to the normalization process based on the citing-side. In the calculation of the SNIP the type of document is not considered and the indicator tends to give higher values for journals with a large number of reviews. The SCImago journal ranking (SJR) (Gonzalez-Pereira et al. 2010) is interesting as it considers the prestige of the citing journals, but as the SNIP the type of document is not taken into account and journals that publish only reviews or publish a high percentage of reviews have the highest values. The SNIP and the SJR consider citations counts for short and fixed citation windows. Short citation windows may not be satisfactory for journals where citation impact matures slowly. If the aim is to predict the impact of a document along the years to use longer and variable citation windows should give better results.

The main goal of this study is to introduce a new indicator, the journal relative impact $(J R I)$, that will be shown to be robust, stable over time, with predictive power and that considers the varying time lag between publication and citation. The JRI is a field normalized indicator that considers a variable citation window and the different type of documents. Not only the number of citations a paper gets varies widely from field to field but also the time lag between publication and citation does also vary. On the development of an indicator for journal assessment we are confronted frequently with the length of the citations window used. Here we have contradictory requirements. An indicator that provides information of the scientific performance of a journal within a short time is desirable and this is only possible using short citation windows, but the consequence is that short time lag areas are better represented than long time lag areas and these may be assessed by a rather small (and possibly not significant) share of the citations they will collect along 
their full lifetime. Journals appear and disappear or change editorial policy, sometimes within short periods. If these are to be assessed by indicators by the ones discussed here, short publication and citation windows must be used. On the other hand, an indicator that considers more information about the citations obtained by a given journal will allow more robust and stable indicators and these indicators will have a higher predictive power. We should also consider that more information about citations will allow a better assessment of the scientific performance of a journal classified in several subject categories. This is only possible using long and variable citation windows. From our point of view we consider that an indicator with these features is important. However, we know that the introduction of this type of citation windows industrially is a little more difficult and implies the disadvantages of the longer citation windows.

As mentioned earlier the type of document is also taken into account in the normalization process proposed here. The consideration of the type of documents used depends on its yield of citations and not on any preconceived idea of the citation relevance that would be impossible to generalize.

It should be stressed that we propose the use of variable citation windows in the sense that we associate a certain window to each subject category so that the citations counted represent an expected percentage (about 18\%) of the citations to be obtained in the total lifetime of the publications. The rationale for this comes from the observation that the mean number of citations per document evolves in time in different ways. The technique proposes here provides the equivalent of a fair comparison of the total citation count obtained by the journal.

In the following sections the methodological aspects, the results and discussion of the application of this indicator are presented.

\section{Methodology}

Web of Science (WoS) data are used throughout in all numerical applications presented in this document.

The impact of different types of documents depends on the scientific area. In this work, within a given subject category, we considered all the documents published in a given year, in journals classified in that subject category and counted the total number of citations obtained in a 5 year period. For each document type we determined the fraction of citations obtained by these documents to the total number of citations. Then, only those types of documents that obtained $5 \%$ or more of the total citations were considered in the calculation of the indicator. The only exception is for journals that only publish review articles and are classified in a subject category where review articles get less than $5 \%$ of all citations.

Using larger citations window for counting publications and citations improves the statistics, but hinders the up to date assessment of the performance of the journal. In this paper publications are considered for a 5 year period. The time lag between publication and citations varies widely among scientific areas. To account this, a variable citation window will be used. If the same 5 years used to count documents were used to observe citations, the number of citations counted would be relatively small for some subject categories. For Chemical, Engineering, for example, only $12 \%$ of the citations obtained in the 19 years life span of the documents are collected in the initial 5 year citation window. A similarly low figure of $10 \%$ is obtained for Computer Science, Interdisciplinary Applications. The compromise proposal is to adjust the citation window to a minimum of $18 \%$ of the expected citations for the entire life of the documents, here taken to be 
19 years. A detailed justification for this criterion is discussed in "Definition of the citation window" section. It should be noticed that no comparable citations data exists today for longer periods.

We now introduce the new indicator, the $J R I$, as the value of the average impact (citations per document) of the documents published in that journal relative to the average impact of the same type of documents published in all journals that are grouped by the WoS in a certain subject category. In simple words, the $J R I$ is the ratio of the actual to the expected citations.

Consider that a journal, $a$, in a certain period, $y$, has published $n_{a}$ documents and that this journal belongs to the subject categories $i=\{1,2 \ldots N\}$, having each subject category $\alpha$ journals. Consider that, in a given period, $x$, these documents obtained $C_{y x a}$ citations. We define now a procedure of normalization of the number of citations. The JRI of journal $a$ as seen within subject category $i$ is given by:

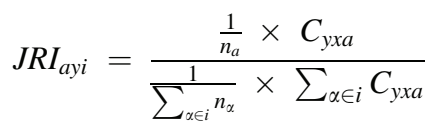

The summation $\sum_{\alpha \in i} n_{\alpha}$ represents the total number of documents published in all journals of subcategory $i$ during the period $y$ (cited years) and the summation $\sum_{\alpha \in i} C_{y x a}$ represents the citations that these documents obtained in period $x$ (citing years). Here $y$ is equal to 5 years for all journals and $x$ is larger than or equal to 5 years this value being dependent on the subject category. If $x$ is 5 years, then $x$ and $y$ refer to the same period. If $x$ is 6 years, then citations are counted during the period $y$ with an extra year.

In more general terms a given journal may belong to more than one subject category in the WoS classification. Consider now a journal that belongs to "1" and "2" subject categories and that it obtains a high $J R I_{a y l}$ for the " 1 " subject category but not a so high $J R I_{a y 2}$ for the " 2 " subject category. How should this journal be assessed in relation to these disparate values of the $J R I_{a y}$ ?

We can consider three different situations for a journal belonging to subject categories "1" and "2".

(1) When this journal publishes documents within subject category " 1 " those documents will be considered by the scientific community " 1 " only;

(2) When this journal publishes documents within subject category "2" those documents will be considered by the scientific community " 2 " only;

(3) When this journal publishes documents with content related with both categories " 1 " and " 2 " (multidisciplinary documents), those documents will be considered by the scientific community of both subject categories.

For simplicity, assume that, of the $n_{a}$ documents in journal $a, \frac{n_{a}}{N}$ documents will be considered by each of the $N$ scientific communities associated with one of the subject categories as their own. The expected number of citations obtained by these $\frac{n_{a}}{N}$ documents is:

$$
\begin{gathered}
\frac{n_{a}}{N} \times\left(\frac{1}{\sum_{\alpha \in i} n_{a}} \times \sum_{\alpha \in i} C_{y x a}\right)=\frac{n_{a}}{N} \times \frac{\frac{1}{n_{a}} \times C_{y x a}}{J R I_{a y i}} \\
\frac{C_{y x a}}{N} \times \frac{1}{J R I_{a y i}}
\end{gathered}
$$

The total of the expected citations is then obtained as the summation of these expected citations over all $N$ subject categories: 


$$
\frac{C_{y x a}}{N} \times \sum_{i=1}^{N} \frac{1}{J R I_{a y i}}
$$

The journal relative impact is defined as the ratio of the actual number of citations that are observed to the expected citations:

$$
\begin{gathered}
J R I_{a y}=\frac{C_{y x a}}{\frac{C_{y x a}}{N} \times \sum_{i=1}^{N} \frac{1}{J R I_{a y i}}} \\
\frac{1}{J R I_{a y}}=\frac{1}{N} \sum_{i=1}^{N} \frac{1}{J R I_{a y i}}
\end{gathered}
$$

This averaging procedure (harmonic mean), depends on the hypothesis that a journal included by the WoS in several subject categories publishes documents that can be identified as belonging to just one of the subject categories and that they are evenly distributed among them. In reality it is fair to assume that some documents are truly multidisciplinary and are thus identified by several scientific communities (associated with different subject categories) as their own with the consequence that are likely to be cited by a wider public. This being the case, the number of expected citations will be larger than that estimated above for each share of documents (Eq. 3 above) and the expected total number of citations will be larger than that given by Eq. 4 . The final conclusion is that the actual $J R I_{a y}$ is smaller than the result given by Eq. 6 above. In this argument, we disregard the case of a multidisciplinary publication accepting documents that would not be considered up to publication standards in the other subject categories as all journals classified as multidisciplinary by the WoS that are, normally, of very high selectivity.

In order to clarify the readers, the calculation of the JRI for the journal Langmuir is presented below. The number of documents (article, review, proceedings paper, correction, editorial material and biographical-item) published in Langmuir between 2003 and 2007 was 8,791 . The journal belongs to three subject categories [Chemistry, Multidisciplinary (CM); Chemistry, Physical (CP) and Materials Science Multidisciplinary (MSM)].

Step 1

For the subject category Material Sciences Multidisciplinary, the total number of citations in a 5 year period of the documents published in 2003 in all journals classified in this subject category was determined. The contribution of each type of document for the total number of citations is then determined and we select those types contributing with a percentage of $5 \%$ or more of the total number of citations. For this subject category, articles, proceedings paper and reviews have the highest percentage as shown in Table 1.

Step 2

After the selection of those types of documents that will be considered in the calculation of the $J R I_{a y i}$, for this subject category, the length of the citation window was determined. For all articles, proceedings papers and reviews published in journals that belong to Material Sciences Multidisciplinary the number of citations received in each year after publication was determined. This was done for the documents published between 1990 and 2008. The total number of citations received by the documents published between 1990 and 1994 (as we are considering 5 years of publications) using a 5, 6, 7 years and so on citation window 
Table 1 Number of citations obtained between 2003 and 2007 by the documents published in 2003 in journals that belong to the subject category Material Sciences Multidisciplinary. Those type of documents with more than $5 \%$ of the total citations are in bold

\begin{tabular}{lrrr}
\hline Type of document & Documents (2003) & Citations (2003-2007) & \% of the total citations \\
\hline Article & 27,244 & 278,683 & $\mathbf{8 1 . 0 6}$ \\
Bibliography & 5 & 8 & 0.00 \\
Biographical-item & 54 & 27 & 0.01 \\
Book review & 2 & 2 & 0.00 \\
Correction & 162 & 81 & 0.02 \\
Editorial material & 769 & 1,551 & 0.45 \\
Letter & 421 & 1,414 & 0.41 \\
Meeting abstract & 16 & 1 & 0.00 \\
News item & 683 & 579 & 0.17 \\
Proceedings paper & 7,563 & 42,606 & $\mathbf{1 2 . 3 9}$ \\
Reprint & 10 & 21 & 0.01 \\
Review & 476 & 18,828 & $\mathbf{5 . 4 8}$ \\
Total & 37,405 & 343,801 & 100.00 \\
\hline
\end{tabular}

Table 2 Distribution of the citations received by the articles, proceedings paper and reviews published in journals that belong to the subject category Material Sciences, Multidisciplinary

\begin{tabular}{|c|c|c|c|c|c|c|c|c|c|c|}
\hline \multirow{2}{*}{$\begin{array}{l}\text { Publication } \\
\text { year }\end{array}$} & \multicolumn{10}{|c|}{ Citations received in each year } \\
\hline & 1990 & 1991 & 1992 & 1993 & 1994 & 1995 & 1996 & $\ldots$ & 2007 & 2008 \\
\hline 1990 & 1,099 & 6,314 & 10,094 & 9,663 & 8,845 & 8,501 & 7,969 & $\ldots$ & 4,951 & 4,956 \\
\hline 1991 & & 1,322 & 8,241 & 11,920 & 11,981 & 11,325 & 10,782 & $\ldots$ & 6,851 & 6,852 \\
\hline 1992 & & & 1,950 & 9,698 & 14,379 & 14,600 & 13,669 & $\ldots$ & 8,969 & 9,501 \\
\hline 1993 & & & & 2,012 & 10,093 & 15,331 & 15,617 & $\cdots$ & 9,325 & 9,956 \\
\hline 1994 & & & & & 1,704 & 9,949 & 16,458 & $\ldots$ & 10,986 & 11,230 \\
\hline 1995 & & & & & & 1,432 & 8,143 & $\ldots$ & 8,334 & 8,588 \\
\hline$\cdots$ & $\cdots$ & $\cdots$ & $\cdots$ & $\cdots$ & $\cdots$ & $\cdots$ & $\cdots$ & $\cdots$ & $\cdots$ & $\cdots$ \\
\hline 2007 & & & & & & & & $\ldots$ & 9,315 & 70,871 \\
\hline 2008 & & & & & & & & & & 12,178 \\
\hline YAP & 0 & 1 & 2 & 3 & 4 & 5 & 6 & $\ldots$ & 17 & 18 \\
\hline $\mathrm{TC}$ & & & & & 109,315 & 169,021 & 233,516 & $\ldots$ & 768,568 & 811,063 \\
\hline $\begin{array}{l}\% \text { of the } \\
\text { total } \\
\text { citations }\end{array}$ & & & & & 13.5 & 20.8 & 28.8 & $\ldots$ & 94.8 & 100.0 \\
\hline
\end{tabular}

YAP Year after publication

TC Total of citations obtained by the documents published between 1990 and 1994

was determined. These citations were compared with the total expected for the entire life of the documents, here taken to be 19 years. A minimum of $18 \%$ of the total is required. For Material Sciences, Multidisciplinary, the length of the resultant citations window is 6 years. The percentage of the total citations obtained for 6 years is represented in bold in Table 2. In Table 2 the data used to define the citations window is presented. 
Table 3 Calculation of the $J R I_{\text {ayi }}$ and $J R I$ for the journal Langmuir considering the documents published between 2003 and 2007

\begin{tabular}{|c|c|c|c|c|c|c|}
\hline $\begin{array}{l}\text { Subject } \\
\text { category }\end{array}$ & $\begin{array}{l}\text { Type of } \\
\text { documents }\end{array}$ & $\begin{array}{l}\text { Length of } \\
\text { the citation } \\
\text { window (years) }\end{array}$ & $\begin{array}{l}\text { Mean number of } \\
\text { citations per } \\
\text { document for } \\
\text { the subject category }\end{array}$ & $\begin{array}{l}\text { Mean number } \\
\text { of citations per } \\
\text { document for } \\
\text { the journal }\end{array}$ & $J R I_{a i}$ & $J R I$ \\
\hline $\mathrm{CM}$ & Article; review & 5 & 6.366 & 7.612 & 1.196 & 1.506 \\
\hline $\mathrm{CP}$ & $\begin{array}{l}\text { Article; review; } \\
\text { proceeding papers }\end{array}$ & 5 & 4.949 & 7.607 & 1.537 & \\
\hline MSM & $\begin{array}{l}\text { Article; review; } \\
\text { proceeding papers }\end{array}$ & 6 & 6.035 & 11.955 & 1.981 & \\
\hline
\end{tabular}

In step 1 and 2 the procedure used in the selection of the type of documents and the length of the citations window was only presented for Material Sciences, Multidisciplinary. The same procedure was applied for the other subject categories that the journal Langmuir belongs to.

Step 3

The values of the $J R I_{a y i}$ and the $J R I$ for the journal Langmuir determined by Eqs. 1 and 6, respectively, are presented in Table 3. The type of documents considered within each subject category, the length of the citation window, the mean number of citations per document for each subject category and the mean number of citations per document for the journal are also presented.

In the next section the indicator proposed was calculated for a set of journals in order to discuss the features presented by the JRI.

\section{Results and discussion}

Definition of the citation window

Short citations window are normally used for the development of impact indicators. For the definition of the $S J R$, the authors stated that a 3 year citation window was chosen because the citation peaks of a significant number of journals in Scopus are reached within this period (Gonzalez-Pereira et al. 2010). In fact, each scientific field has its own time lag to reach the citation peak. In some fields this happens more slowly than in others and the average number of citations per document is also different. However, we should not forget the behaviour of these documents after this peak of citations if we want to use an indicator that allows predicting the impact in the future. In order to illustrate how the mean number of citations per document decreases in time we studied the variation of the average number of citations per document, for two subject categories, each year after publication up to the 18th year.

In Fig. 1, the evolution of the average number of citations per document suggests that this type of study should consider a much longer period as the tail after the 18th year may be thought to contribute significantly to the aggregate of citations. However, we should take into account that publication and citation habits have changed significantly in the last few years. Researchers publish and cite more now than in the past. On the other hand, the coverage of journals by the databases usually used in bibliometric studies has been increased over time and very especially in the last few years. These aspects may introduce some noise on the analysis of the evolution of the average number of citations per document. 

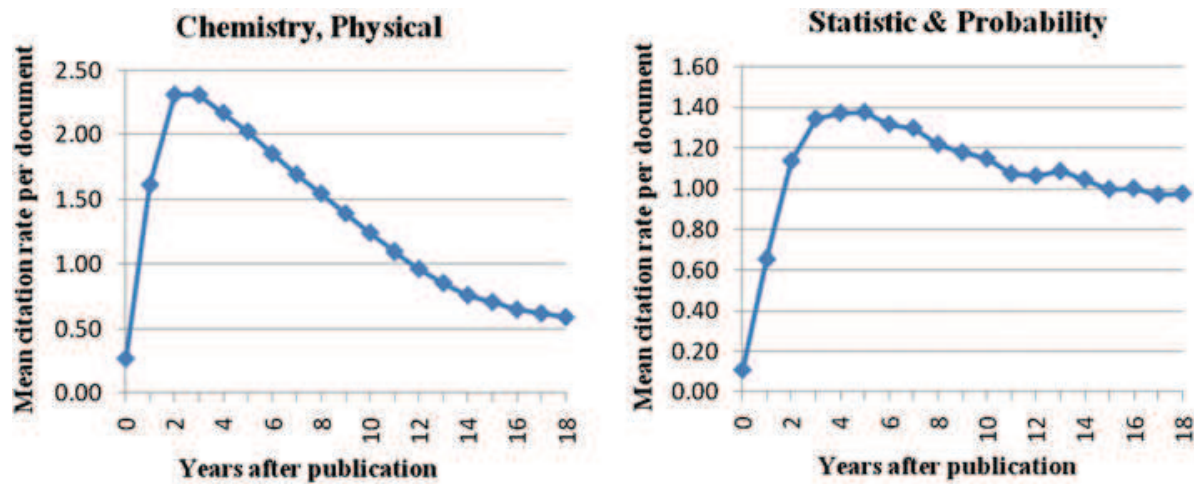

Fig. 1 Evolution of the average number of citations per document in Chemistry, Physical and Statistic \& Probability for the documents published between 1990 and 2008

For the journals classified in the WoS in Chemistry, Physical and Statistic \& Probability the number of documents published from 1990 to 2008 and the number of citations obtained by these documents in each year after publication were counted. For example the average number of citations per document obtained in the fourth year after publication was calculated considering the number of citations obtained in that year by the documents published in the period 1990-2004. In Fig. 1 we can observe that for Chemistry, Physical the citations peak is reached between the second and the third year after publication and the value is 2.3. For Statistic \& Probability the citations peak happens between the fourth and fifth year after publication with a peak value of about 1.40. If we look for the behaviour of these documents after the citation peak we can observe a faster decrease of the mean citation per document for Chemistry, Physical than for Statistic \& Probability. For both subject categories the mean citation per document obtained for the 18th year is still high. In part these values may be explained by the fact that we are counting citations in 2008 for documents published in 1990. As the number of journals indexed in the WoS increased in the last few years, citations counts for more recent years are based in a larger set of journals and this may explain the high values for the mean citation per document. Taking into account the behaviour of the mean citation per document over time for the set of documents presented above we defined a citation window that allows an estimation of the mean citation per document for a set of documents in a distant future. The percentage of citations used in the definition of the citation window length was about $18 \%$. In order to establish this value the ratio between the total number of citations for a 5 years citation window and the total number of citations for a 19 years citation window was determined. The lowest percentage was chosen so that neither of the subject categories gets this percentage in less than 5 years. It may be questionable why a higher percentage is not used. In fact the use of a higher percentage might allow better results for the indicator, but, it would be necessary to work with citation windows too long for some subject categories. This implies that a given journal must be indexed in the database during several years before the first value for the JRI can be calculated. For example if a journal that was indexed in 2010 in the database and that belongs to a subject category where the citation window was defined at 8 years, the first value of the JRI for this journal would be only possible in 2018 .

To use an indicator that considers short citation windows, as the SNIP or the $S J R$, does not imply indexing the journal in the database for many years before getting the first value for the indicator. However, if the aim is to predict the future mean citations per document 

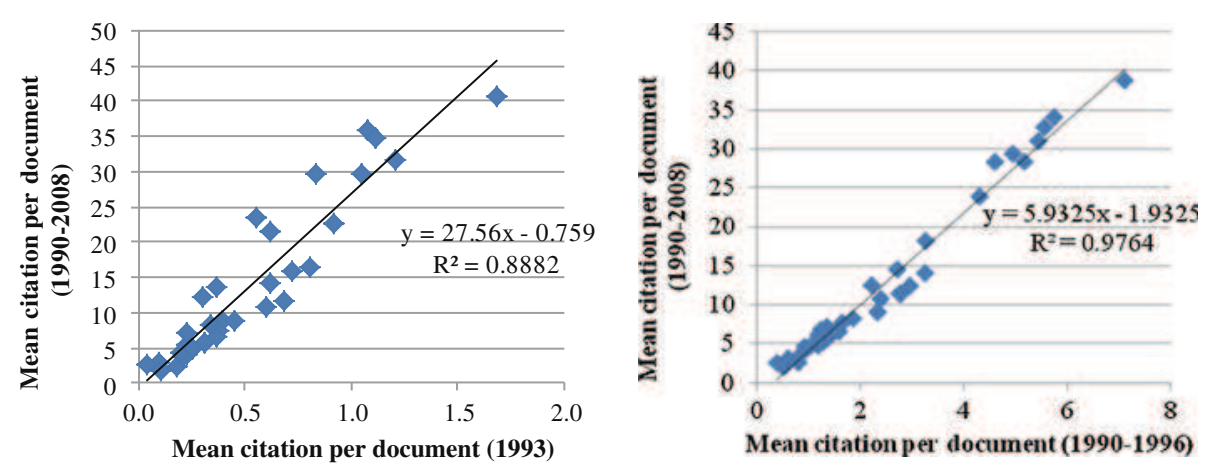

Fig. 2 Influence of the length of the citation window on the predictability of the mean number of citations per document in a period of 19 years for a set of journals. On the left, documents published in 1990-1992 are considered while on the right we take documents published in 1990-1994

for a set of documents, considering the results obtained in the present, to use a citation window defined as function of the culture of citation of the scientific community associated with each subject category may give better results.

For a set of journals that belong to the subject category Statistic \& Probability, we determined the relation between the mean of the number of citations (in 1993) per document published between 1990 and 1992 and the mean of the number of citations (in the period 1990 and 2008) per document for these same documents published between 1990 and 1992. This is plotted on the left hand side of Fig. 2. For the same set of journals, we determine now the relation between the mean number of citations (in 1990-1996) per document published between 1990 and 1994 and the mean number of citations (in the period 1990 and 2008) per document for these same documents published between 1990 and 1994. This is shown on the right hand side of Fig. 2.

The final goal of a journal impact indicator is to predict future citations of the documents published in a particular journal. This study confirms that the observation of citations in one single year (as done for $S N I P$ and $S J R$ ), leads to a lower predictability than taking a longer observation window as in the proposed JRI indicator. For SNIP and SJR, citations in a particular year are counted for documents published in previous years and this may include the citations peak of some documents but not for others. The citations peak varies from one scientific area to another and the citation decay curve varies more widely making a short time lag a lower predictor of the longer term behaviour.

Differences between fixed and variable citation windows

Fixed citation windows are normally used independently of the evolution of the citations over time in different fields. As mentioned earlier this different evolution influences the results given by the performance indicators (Moed et al. 1998). In this paper we propose to use a longer and a variable citation window between subject categories instead of fixed citation windows.

Imagine a journal A classified in subject category S1 and S2. The content of the journal is of more interest for the scientific community of S1 than for S2 and then the impact of the journal results, essentially, from the impact caused in S1.

For each of these subject categories was observed the aspects pointed out below (Table 4).

\section{旬 Springer}


Table 4 Length of the citation windows for $\mathrm{S} 1$ and $\mathrm{S} 2$

\begin{tabular}{ll}
\hline Subject category & $\begin{array}{l}\text { Length of the citation windows } \\
\text { (18\% of the total citations) }\end{array}$ \\
\hline S1 & 6 \\
S2 & 5 \\
\hline
\end{tabular}

The relation between the expected average number of citations per document for $\mathrm{S} 1$ and $\mathrm{S} 2$ using a 5 and 6 citation windows is:

$$
\begin{aligned}
& {\left[\frac{1}{\sum_{\alpha \in \mathrm{S} 2} n_{\alpha}} \times \sum_{\alpha \in \mathrm{S} 2} C_{y x a}\right]_{x=6}>\left[\frac{1}{\sum_{\alpha \in \mathrm{S} 1} n_{\alpha}} \times \sum_{\alpha \in \mathrm{S} 1} C_{y x a}\right]_{x=6}} \\
& {\left[\frac{1}{\sum_{\alpha \in \mathrm{S} 2} n_{\alpha}} \times \sum_{\alpha \in \mathrm{S} 2} C_{y x a}\right]_{x=5}>\left[\frac{1}{\sum_{\alpha \in \mathrm{S} 1} n_{\alpha}} \times \sum_{\alpha \in \mathrm{S} 1} C_{y x a}\right]_{x=5}}
\end{aligned}
$$

The relation between the increase of the average number of citations per document for $\mathrm{S} 1$ and S2 when we go from a citation window of 5-6 years is:

$$
\frac{\left[\frac{1}{\sum_{\alpha \in \mathrm{S} 1} n_{\alpha}} \times \sum_{\alpha \in \mathrm{S} 1} C_{y x a}\right]_{x=6}}{\left[\frac{1}{\sum_{\alpha \in \mathrm{S} 1} n_{\alpha}} \times \sum_{\alpha \in \mathrm{S} 1} C_{y x a}\right]_{x=5}}>\frac{\left[\frac{1}{\sum_{\alpha \in \mathrm{S} 2} n_{\alpha}} \times \sum_{\alpha \in \mathrm{S} 2} C_{y x a}\right]_{x=6}}{\left[\frac{1}{\sum_{\alpha \in \mathrm{S} 2} n_{\alpha}} \times \sum_{\alpha \in \mathrm{S} 2} C_{y x a}\right]_{x=5}}
$$

The relation between the ratio of the expected average number of citations per document for $\mathrm{S} 1$ and for $\mathrm{S} 2$ when we go from a citation window of 5 years to 6 years is:

$$
\frac{\left[\sum_{\alpha \in S 2} n_{\alpha}\right.}{\left[\frac{1}{\sum_{\alpha \in S 1} n_{\alpha}} \times \sum_{\alpha \in S 2} C_{y \in S 1} C_{y x a}\right]_{x=6}}>\frac{\left[\frac{1}{\sum_{\alpha \in S 2} n_{\alpha}} \times \sum_{\alpha \in S 2} C_{y x a}\right]_{x=5}}{\left[\frac{1}{\sum_{\alpha \in S 1} n_{\alpha}} \times \sum_{\alpha \in S 1} C_{y x a}\right]_{x=5}}
$$

Using a 5 year citation windows for both subject categories, as the content of the journal is of more interest for $\mathrm{S} 1$, it is expected that:

$$
\left[J R I_{y \mathrm{~S} 1}\right]_{x=5}>\left[J R I_{y \mathrm{~S} 2}\right]_{x=5} .
$$

The $\left[J R I_{y \mathrm{~S} 1}\right]_{x=5}$ will also be influenced by citations that come from the scientific community of $\mathrm{S} 2$ overestimating the scientific performance of the journal in relation to $\mathrm{S} 1$.

This same behaviour is expected when a 6 year citation windows is used for both subject categories. For this length window the numerator and the denominator in the JRI ayi equation will be higher than for a 5 year citation window, but the denominator for the $\left[J R I_{y \mathrm{~S} 1}\right]_{x=6}$ will increase more than the denominator for the $\left[J R I_{y \mathrm{~S} 2}\right]_{x=6}$ in relation to the 5 year citation window if we consider the inequality (9).

Using a 6 year citation window the overestimation of the $\left[J R I_{y \mathrm{~S} 1}\right]_{x=6}$ by the citations that come from the scientific community of S2 will be attenuated by the considerable increase of the average number of citations per document observed in S1. Then it is expected that:

$$
\left[J R I_{y \mathrm{~S} 1}\right]_{x=5}>\left[J R I_{y \mathrm{~S} 1}\right]_{x=6}
$$

The inequality (10) allows concluding that: 


$$
\left[J R I_{y \mathrm{~S} 2}\right]_{x=6}<\left[J R I_{y \mathrm{~S} 2}\right]_{x=5}
$$

The comparison of the $J R I_{\text {ayi }}$ using different citations windows suggests that:

$$
\left[J R I_{y \mathrm{~S} 2}\right]_{x=6}<\left[J R I_{y \mathrm{~S} 2}\right]_{x=5}<\left[J R I_{y \mathrm{~S} 1}\right]_{x=6}<\left[J R I_{y \mathrm{~S} 1}\right]_{x=5}
$$

This shows that the JRI indicator of a journal belonging to a low citation subject category S1, may be overestimated by the citations that come from the marginal presence (and the consequent classification) of some material from a very high citation subject category S2 when short and fixed citation windows are used. On the other hand, the JRI may be underlie depressed by the presence of this material if longer and fixed citations windows are used. These overestimated and depressing effects are lower if variable length citation windows are used as suggested here. In fact the harmonic averaging process makes the result very dependent on the lowest value, and in the example above, the journal A would obtain a $J R I$ value close to that associated with its performance as assessed from the point of view of subject category S2.

The differences in the scientific performance of journals for fixed and variable citation windows are presented below.

In order to show the differences between the use of fixed and variable citation windows the JRI was determined for a set of journals that belong to the Chemistry, Physical (CP); Engineering, Chemical (EC); Chemistry, Applied (CA); Chemistry, Multidisciplinary (CM) and Materials Science, Multidisciplinary (MSM) subject categories. The JRI was determined using a fixed citation window of 3 years and then using a variable citation window (5 years for CP and CM and 6 years for MSM, CA and EC). In Table 5, the results obtained for the $J R I_{\text {ayi }}$ and the $J R I$ are presented.

In Table 5 we can see that the use of a variable citation window shows a different scenario of the scientific performance of the journals in relation to the fixed citation window. Several situations can be observed when a variable citation window is applied:

(1) The $J R I_{a y i}$ obtained for one of the subject categories that the journal belongs to, is lower than that obtained using a fixed citation window, but for the other subject category is higher than that obtained using a fixed citation window. This is the case of the Journal of Catalysis and Langmuir.

(2) The $J R I_{a y i}$ obtained for each of the subject categories that a given journal belongs to is higher than that obtained using a fixed citation window. This was observed for Catalysis Today.

(3) The $J R I_{a y i}$ obtained for each of the subject categories that a given journal belongs to is lower than that obtained using a fixed citation window. The journal Topics in Catalysis and the journal Organic Process Research \& Development are examples.

The Journal of Catalysis has a very large $J R I_{a y}$ for Engineering, Chemical than for Chemistry, Physical in both types of citation windows, but for a variable citation window the $J R I_{a y i}$ is lower than for a fixed citation window for Engineering, Chemical. This suggests that the documents published in this journal are mainly related with the subject category Chemistry, Physical and this causes an overestimation of the $J R I_{a y i}$ determined for Engineering, Chemical due to the higher average number of citations per document of Chemistry, Physical. The difference between the average number of citations per document for these two subject categories is higher for a fixed citation window and this may explain why the $J R I_{\text {ayi }}$ is higher for Engineering, Chemical when a fixed citation window is used. 
Table 5 Values obtained for the $J R I$ using a fixed and a variable citations window

\begin{tabular}{|c|c|c|c|c|c|c|}
\hline \multirow[t]{2}{*}{ Journal title } & \multirow[t]{2}{*}{ Subject category } & \multicolumn{2}{|l|}{$J R I_{a y i}$} & \multicolumn{2}{|c|}{$\begin{array}{l}\text { Average number of citations per } \\
\text { document }\end{array}$} & \multirow{2}{*}{$\begin{array}{l}J R I \\
\text { Variable } \\
\text { citation } \\
\text { window }\end{array}$} \\
\hline & & $\begin{array}{l}\text { Fixed } \\
\text { citation } \\
\text { window }\end{array}$ & $\begin{array}{l}\text { Variable } \\
\text { citation } \\
\text { window }\end{array}$ & $\begin{array}{l}\text { Fixed } \\
\text { citation } \\
\text { window }\end{array}$ & $\begin{array}{l}\text { Variable } \\
\text { citation } \\
\text { window }\end{array}$ & \\
\hline \multirow[t]{2}{*}{$\begin{array}{l}\text { Journal of } \\
\text { Catalysis }\end{array}$} & $\begin{array}{l}\text { Engineering, } \\
\text { chemical }\end{array}$ & 4.200 & 3.812 & 1.094 & 3.976 & \multirow[t]{2}{*}{2.619} \\
\hline & Chemistry, physical & 1.782 & 1.995 & 2.578 & 4.949 & \\
\hline \multirow[t]{3}{*}{ Catalysis Today } & Chemistry applied & 1.309 & 1.580 & 1.407 & 5.120 & \multirow[t]{3}{*}{1.395} \\
\hline & $\begin{array}{l}\text { Engineering, } \\
\text { chemical }\end{array}$ & 1.725 & 2.034 & 1.068 & 3.976 & \\
\hline & Chemistry, physical & 0.789 & 0.975 & 2.336 & 4.949 & \\
\hline \multirow[t]{3}{*}{ Langmuir } & $\begin{array}{l}\text { Chemistry, } \\
\text { multidisciplinary }\end{array}$ & 1.172 & 1.196 & 3.056 & 6.366 & \multirow[t]{3}{*}{1.506} \\
\hline & Chemistry, physical & 1.535 & 1.537 & 2.336 & 4.949 & \\
\hline & $\begin{array}{l}\text { Materials science, } \\
\text { multidisciplinary }\end{array}$ & 2.109 & 1.981 & 1.700 & 6.035 & \\
\hline \multirow{2}{*}{$\begin{array}{l}\text { Organic Process } \\
\text { Research \& } \\
\text { Development }\end{array}$} & Chemistry, applied & 1.351 & 1.063 & 1.407 & 5.120 & \multirow[t]{2}{*}{0.874} \\
\hline & Chemistry, organic & 0.764 & 0.742 & 2.489 & 4.798 & \\
\hline \multirow{2}{*}{$\begin{array}{l}\text { Topics in } \\
\text { Catalysis }\end{array}$} & Chemistry, applied & 1.633 & 1.300 & 1.407 & 5.120 & \multirow[t]{2}{*}{1.017} \\
\hline & Chemistry, physical & 0.984 & 0.836 & 2.336 & 4.949 & \\
\hline
\end{tabular}

In the calculation of the $J R I$ for a fixed citations window the documents published between 2003 and 2005 were used and the citations were counted in the same period. The $J R I$ for a variable citations window was determined using the documents published between 2003 and 2007

For the journal Langmuir, the results suggest that the documents published have a scientific performance close to that of the documents published in Chemistry, Physical and Chemistry, Multidisciplinary. The highest value of the JRI ${ }_{a y i}$ in Material Science, Multidisciplinary when a fixed citation window is used may be overestimate by the citations received by the scientific community of Chemistry, Physical and Chemistry, Multidisciplinary. For these two subject categories the average number of citations per document is higher than in Material Science, Multidisciplinary when a fixed citation window is used.

The journal Catalysis Today presents higher $J R I_{a y i}$ values for all the subject categories when a variable citation window is used. The results suggest that the documents published in this journal have a scientific performance close to the average of the subject category Chemistry, Applied and Engineering Chemical. The average number of citations per document is higher in Chemistry, Applied than in Chemistry, Physical when a variable citation window is used. For the documents published in this journal the average number of citations per document is below that obtained for the documents published in journals that belong to Chemistry, Physical, but the average number of citations per document is above that obtained by the documents published in journals from Chemistry, Applied even when the average number of citations per document in Chemistry, Applied (5.120) is higher than in Chemistry, Physical (4.949). This shows that the documents published in Catalysis Today receive the citations later and therefore the performance of the journal is better when a variable citation window is used.

If we use a fixed citation window, we can say that the journal Organic Process Research \& Development performs better in Chemistry, Applied than in Chemistry, Organic in both 
cases. For a fixed citation window of 3 years the average number of citations per document is higher for Chemistry, Organic than for Chemistry, Applied. When a variable citation window is used the subject category Chemistry, Applied presents now the highest value. The results suggest that the documents published in Organic Process Research \& Development have a scientific performance near from those documents published in Chemistry, Applied. The citations that come from the scientific community of Chemistry, Organic overestimate the $J R I_{a y i}$ obtained for the subject category Chemistry, Applied when a fixed citation window is used. The same conclusion can be draw for the journal Topics in Catalysis.

Looking at these journals we can say that a variable citation window allows following in a better way the citations behaviour for the documents published in a given journal, especially for those journals that are classified in several subject categories with very different cultures of citation.

The values obtained for the $J R I$ shows that when a journal belongs to two subject categories the $J R I$ will be closer to the lowest $J R I_{\text {ayi }}$. This does not favour those journals that publish documents from two subject categories and one of the $J R I_{a y i}$ is overestimated because the journal publishes work mainly related with another subject category where the documents get usually more citations.

Stability and complementarity

When developing bibliometric indicators, it is important to keep in mind that one single metric is not enough to measure the scientific performance of a given research body. One indicator may give reliable results when applied in a specific context, but this may not happen in another context. The JRI indicator was compared with the $S J R$ and with the $S N I P$ in relation with its stability and the differences obtained when a set of journals are ranked using these different indicators. The SJR and the SNIP are calculated in a very different way and are available in the Scopus database. The coverage of the different fields by the particular database used is taken into account in the SNIP and the SJR attempts at measuring the prestige of each journal in a way that the different prestige of the citing journals is considered. In the following sub sections the stability of the new indicator, as well as its complementarity with other indicators is discussed.

\section{Stability of the indicators}

A good impact indicator for scientific journals should be robust, i.e. not vulnerable to statistical fluctuations of the documents published and of the citations received. This may require a longer observation window that allows for the effect of editorial policy changes or journal circulation changes to be detected with certainty.

In order to study the stability of the new indicator, we compare in Table 6 the statistical parameters (mean, standard deviation and relative standard deviation) of the values of JRI, $S N I P$ and SJR. The SJR and the SNIP were retrieved from Scopus's database for the years 2009, 2008, 2007, 2006 and 2005. The JRI for each journal was calculated for the documents published in five periods, 2000-2004; 2001-2005, 2002-2006 and 2003-2007.

The results in Table 6 allow the conclusion that the new JRI indicator is more stable than the $S J R$ or the $S N I P$ by application of the non-parametric sign test $(P=0.03<0.05)$ (Lloyd 1984).

This may be explained by two effects. First, the JRI uses longer citations window. Counting only those citations obtained by a set of documents in a given year (as in the 


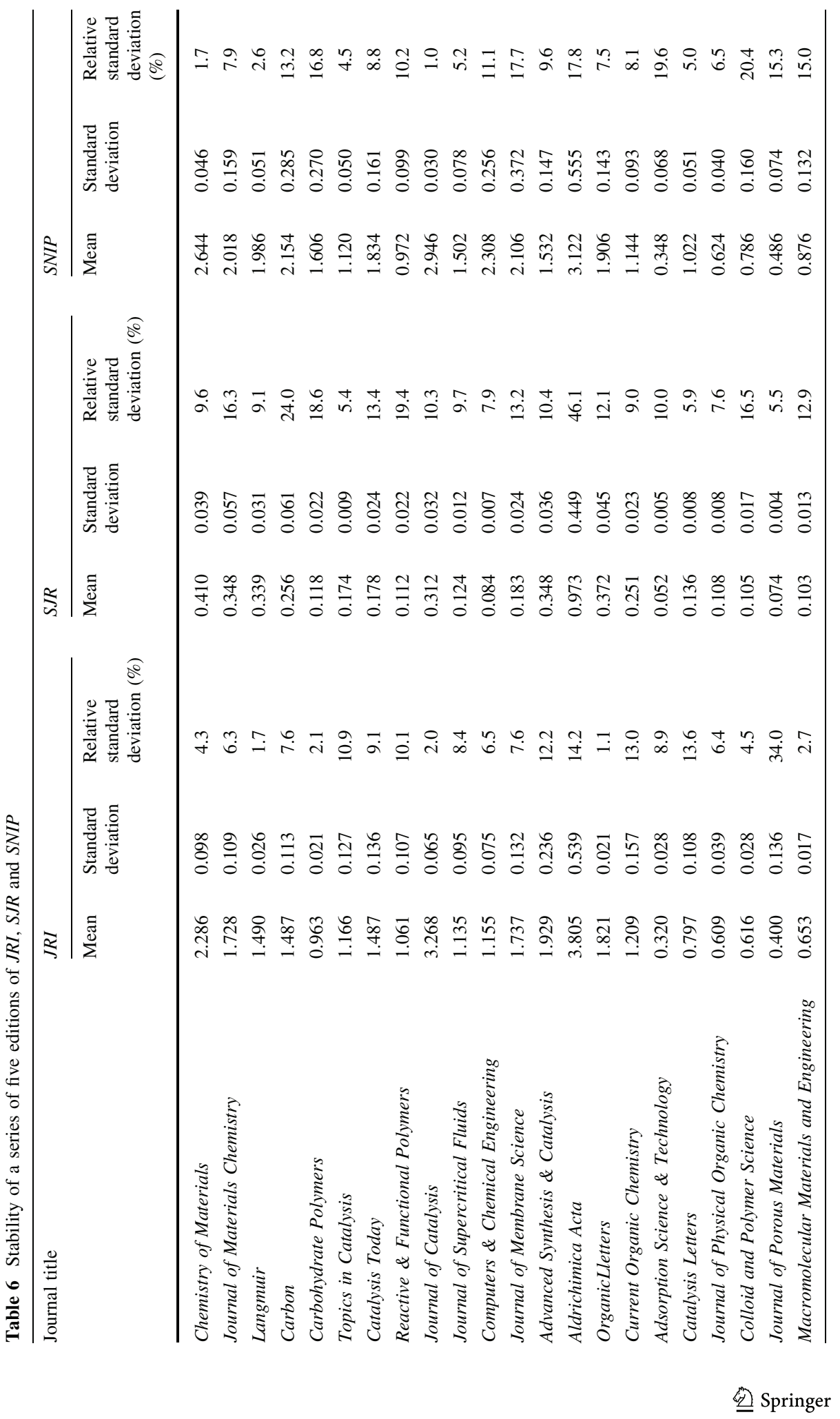




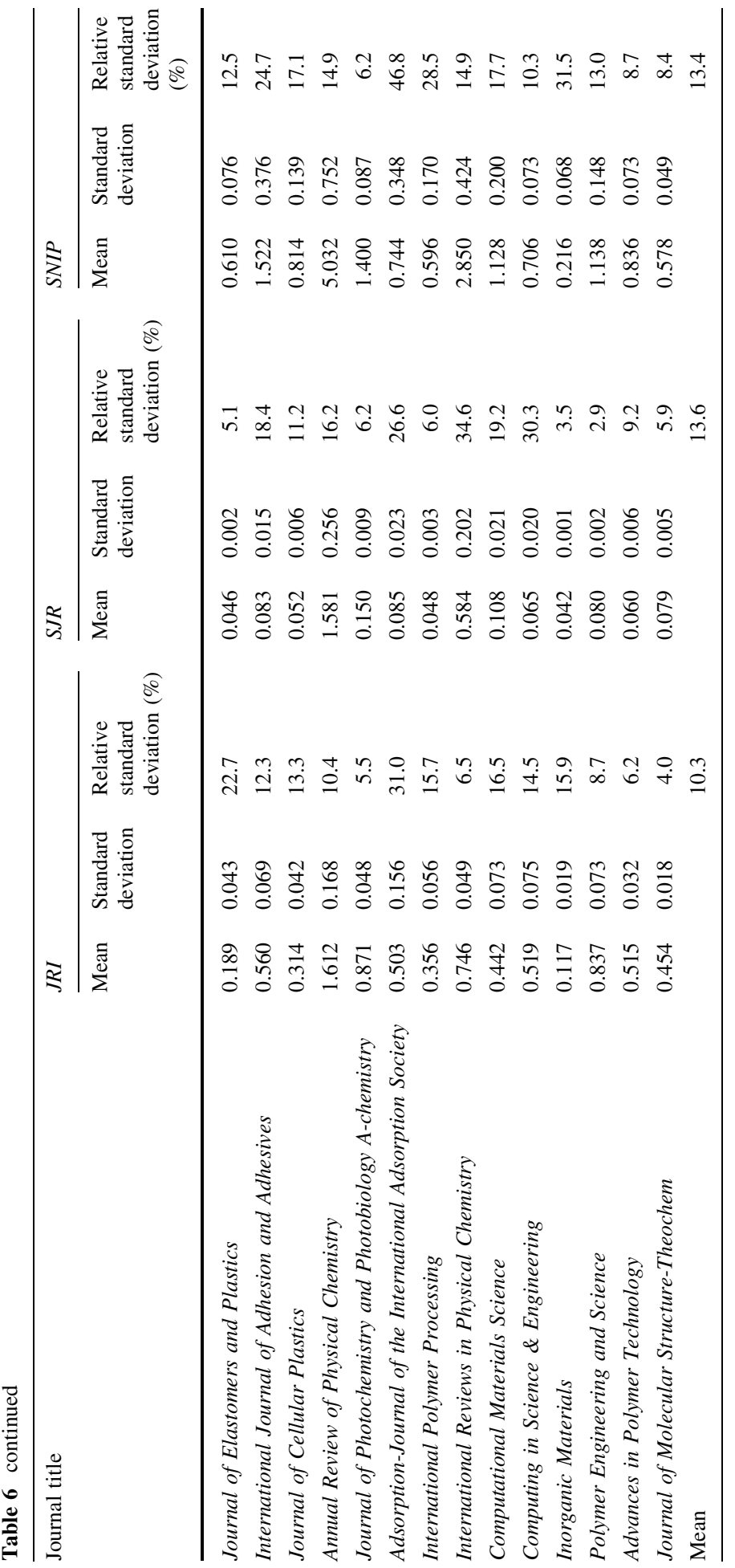

黑 Springer 

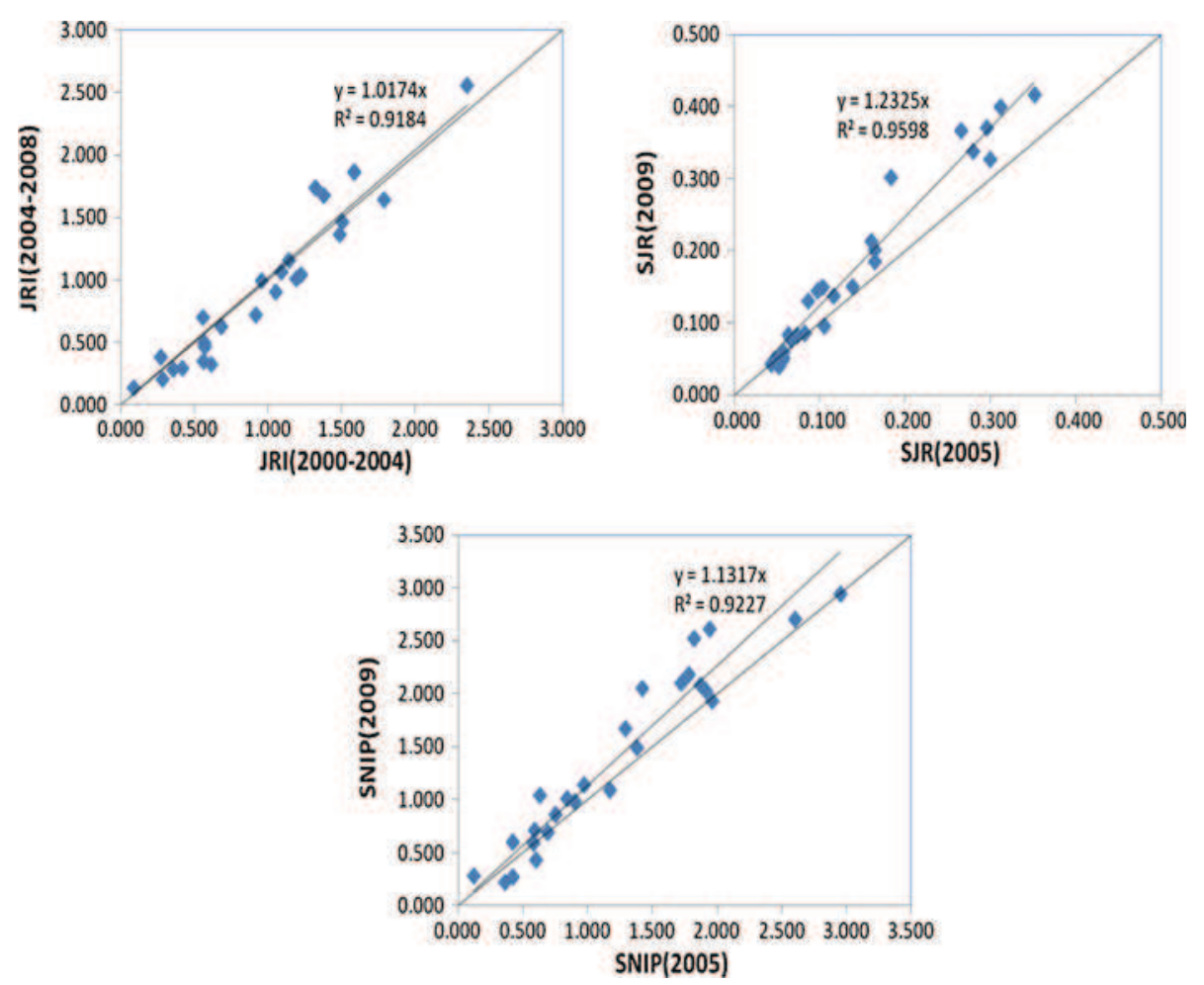

Fig. 3 Values obtained for the JRI, SJR and the SNIP at different times for a set of journals

SNIP and $S J R$ ) the indicator is more vulnerable to yearly fluctuations that influence the citations obtained. When using longer citation windows, features that can influence the citations obtained will tend to more stable averages. Second, a variable citation window is used that allows following the behaviour of the citations of a given journal for the time required for the maturation of citations in the particular subject category that the journal belongs to.

The relation between the $J R I$, the $S J R$ and the $S N I P$ calculated for two different periods is presented in Fig. 3 aiming to appreciate the time evolution of these indicators.

The data in Fig. 3 shows that the SNIP and the SJR suffer from a regular growth over time (13\% for SNIP and $23 \%$ for SJR into 4 year period), while the JRI is rather stable (a $2 \%$ growth was detected well within the statistical uncertainly). This effect is probably due, to a larger extent, to the growth in the last few years of the coverage that the databases used here have adopted. The normalization procedure adopted in the definition of the JRI corrects for this.

Complementarity of the indicators

To appreciate to what extent the JRI, SJR and SNIP indicators give complementary information, we looked at the differences in the journal ranking using these indicators. The SJR and SNIP indicators were retrieved from Scopus database for the year 2005 and the 

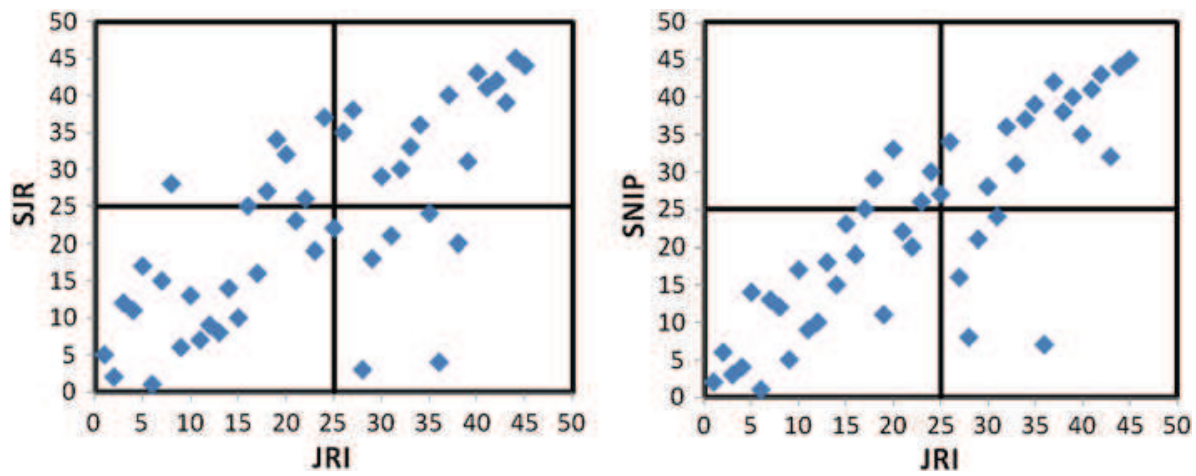

Fig. 4 Differences in the journal rankings when the $J R I$, the $S J R$ and the $S N I P$ are used

$J R I$ was calculated for the documents published between 2000 and 2004. This is shown in Fig. 4.

We see that the rankings can be very different and this is likely to be due to the influence of several parameters. We now discuss the effect of the parameters that suggest that JRI should be preferred.

\section{(1) Type of documents}

Those journals that publish only reviews or a large percentage of reviews are expected to attract a large number of citations. In the calculation of the JRI, the mean number of citations per document of this type of documents is compared with the mean number of citations per document obtained by reviews published in journals that belong to the same subject category. In the calculation of the SJR and the SNIP the type of document is not taken into account and therefore journals that publish only reviews or a large number of reviews will get a better position than in the $J R I$ ranking. This behaviour was observed for the Current Opinion in Colloid \& Interface Science and the International Reviews in Physical Chemistry. The journal Current Opinion in Colloid \& Interface Science is in the 36th position in the JRI ranking while it ranks in the 4th and 7th in the SJR and SNIP rankings, respectively. The journal International Reviews in Physical Chemistry is in the 28th position in the JRI ranking and in the 3th and 8th positions in the SJR and SNIP rankings, respectively.

\section{(2) Citation window}

In the calculation of the $S J R$ a 3 year citation window is used. If a given journal is classified in a subject category that receives a significant number of citations later (after 3 years), this journal will be positioned lower in the SJR ranking than journals that are classified in subject categories that receive a significant number of citations within short period. Using a variable citation window this effect is eliminated as the different time required for the maturation of citations is considered.

\section{(3) Normalization}

The $S J R$ tends to be higher for subject categories where the scientific communities cite more and therefore it is expected that these journals will have a better position in the SJR ranking than those journals that publish work related with subject categories where the authors cite less. The study made by Gonzalez-Pereira et al. 2010) showed that the top ten journals in the subject area of Genetics \& Molecular Biology have a much higher SJR than 
the top ten journals in the subject area of Psychology. The JRI is a field normalized indicator that takes into account the different culture of citations and therefore it is expected that journals classified in subject categories with a high traffic of citations might have a better position in the $S J R$ ranking than in the $J R I$ ranking.

We discuss now the ways in which the SNIP and the SJR indicators can complement the information given by the $J R I$.

(1) The prestige of the citing journal

If a journal receives a large number of citations, but these citations come from journals with low prestige, this journal may be in a better position in the JRI ranking than in the SJR ranking. On the other hand, a journal may receive fewer citations but these citations come from journals with high prestige. In this case the position of the journal in the SJR ranking may be better than in the JRI ranking.

(2) Database citation potential

Moed (2010) defined the SNIP as the ratio between the raw impact per paper (RIP) and the relative database citation potential (RDCP). The RDCP is the ratio between the database citation potential of a given journal $\left(\mathrm{DCP}_{\mathrm{j}}\right)$ and the database citation potential of the journal in the median position in the database $\left(\mathrm{DCP}_{\mathrm{M}}\right)$ relatively to the database citation potential. The effect of the DCP may explain why some journals have a better position in the JRI ranking than in the SNIP ranking while the reverse is observed for others. If a given journal has a high mean number of citations per document and a database citation potential higher than the journal in the median position the SNIP may be lower than the RIP. The JRI for this journal will be high. In this situation it is expected a better position in the JRI ranking than in the SNIP ranking. On the other hand, for a journal with a database citation potential lower than the journal in the median positions the SNIP may be higher than the RIP for a journal with a few citations per document. The $J R I$ for this journal will be low. In this situation it is expected that a journal will have a better position in the SNIP ranking than in the JRI ranking.

Another important point that should not be forgotten is that the SJR and the SNIP are calculated using Scopus data while the JRI uses WoS data. Several studies showed that these databases are different in what concerns to the number of sources indexed and the coverage of these sources over time (Jacso 2005; Lopez-Illescas et al. 2008; Gavel and Iselid 2008). This can also justify some of the differences in the rankings, but we think that it does not influence the results obtained to a great extent. However, the discussion of these results must be considered carefully as we are presenting only tentative conclusions. It will be necessary to analyse in detail the results obtained for the parameters considered in the SNIP and SJR calculation for each journal.

\section{Conclusions}

In this study we introduced the $J R I$ indicator that aims at measuring the scientific performance of a given journal considering the different culture of citation of each subject category. The $J R I$ is a field normalized indicator that uses a variable citation window defined as a function of the time required for the maturation of citations.

The main strengths of the JRI indicator are described below.

- This indicator allows evaluating the scientific performance of a given journal in a better way as it considers the time required for the maturation of citations in each field. This is 
particularly important for those journals that publish works in fields that get a significant number of citations several years after publication.

- For each subject category, we consider the type of documents that generate more than $5 \%$ of the total citations of all documents in that subject category. In this way, the decision to consider a particular document type of a journal depends on its yield of citations and not on any preconceived idea of the citation relevance that is impossible to generalize.

- For journals that belong to several subject categories the scientific performance in each subject category is considered and a certain averaging procedure adopted.

- The JRI presents a significant stability over time and can be used to predict the future behaviour of a given journal.

The comparison of the new indicator with the SJR and the SNIP indicators showed that these indicators should be used together in order to obtain a better description of the scientific performance of a given journal. The JRI, the SJR and the SNIP together allow the assessment of several important aspects of the scientific performance of a given journal taking into account several factors. The JRI was showed to be more appropriate than the $S J R$ and the $S N I P$ for the assessment of the scientific performance of a given journal in the following sense.

- The SNIP and the SJR do not consider in their calculation the type of documents and therefore those journals that publish documents that receive normally a large number of citations (reviews) will be privileged. The JRI compares a journal with others with a similar mix of document types and eliminates the effect of overestimation of the impact by the reviews.

- The $S J R$ use a 3 year fixed citation window privileging those journals that receive a significant number of citations earlier. The JRI considers a variable citation window where the longer times required for the maturation of citations is considered and therefore this effect is eliminated.

- The $J R I$ is a field normalized indicator and allows the comparison of journals from different subject categories. The $S J R$ as stated above tends to be higher for subject categories where the scientific communities cite more. The $S J R$ seems to have some citing-side normalization but this normalization does not appear to be enough to avoid a clear distinction of journals from different fields.

- The JRI presents more stability over time than the SNIP and the SJR. This is an important feature as we can predict the scientific performance of a journal in the present year based on the results obtained in the past year.

Some of the weaknesses of the JRI are:

- The JRI is dependent on the database field's classification.

- The longer citations window used on the calculation of the JRI does not allow providing information about the scientific performance of a journal in a short time especially for those journals that appear and disappear in a short time.

\section{References}

Bergstrom, C. (2007). Eigenfactor: Measuring the value and prestige of scholarly journals. C\&RL News, 68(5), 314-316. 
Bollen, J., Rodriguez, M. A., \& Van De Sompel, H. (2006). Journal status. Scientometrics, 69(3), 669-687.

Bollen, J., \& van de Sompel, H. (2008). Usage impact factor: The effects of sample characteristics on usagebased impact metrics. Journal of the American Society for Information Science and Technology, 59(1), 136-149. doi:10.1002/asi.20746.

Garfield, E., \& Sher, I. H. (1963). New factors in evaluation of scientific literature through citation indexing. American Documentation, 14(3), 195.

Gavel, Y., \& Iselid, L. (2008). Web of Science and Scopus: A journal title overlap study. Online Information Review, 32(1), 8-21. doi:10.1108/14684520810865958.

Glanzel, W., \& Schoepflin, U. (1995). A bibliometric study on aging and reception processes of scientific literature. Journal of Information Science, 21(1), 37-53.

Gonzalez-Pereira, B., Guerrero-Bote, V. P., \& Moya-Anegon, F. (2010). A new approach to the metric of journals' scientific prestige: The SJR indicator. Journal of Informetrics, 4(3), 379-391. doi: 10.1016/j.joi.2010.03.002.

Jacso, P. (2005). As we may search-comparison of major features of the Web of Science, Scopus, and Google scholar citation-based and citation-enhanced databases. Current Science, 89(9), 1537-1547.

Kyvik, S. (2003). Changing trends in publishing behaviour among university faculty, 1980-2000. Scientometrics, 58(1), 35-48.

Leydesdorff, L. (2007). Betweenness centrality as an indicator of the interdisciplinarity of scientific journals. Journal of the American Society for Information Science and Technology, 58(9), 1303-1319. doi: 10.1002/asi.20614.

Lisee, C., Lariviere, V., \& Archambault, E. (2008). Conference proceedings as a source of scientific information: A bibliometric analysis. Journal of the American Society for Information Science and Technology, 59(11), 1776-1784. doi:10.1002/asi.20888.

Lloyd, E. (1984).Handbook of applicable mathematics vol. VI, Part B. Statistics (1st ed.). New York: John Wiley \& Sons.

Lopez-Illescas, C., de Moya-Anegon, F., \& Moed, H. F. (2008). Coverage and citation impact of oncological journals in the Web of Science and Scopus. Journal of Informetrics, 2(4), 304-316. doi: 10.1016/j.joi.2008.08.001.

Moed, H. F. (2010). Measuring contextual citation impact of scientific journals. Journal of Informetrics, 4(3), 265-277. doi:10.1016/j.joi.2010.01.002.

Moed, H. F., Van Leeuwen, T. N., \& Reedijk, J. (1998). A new classification system to describe the ageing of scientific journals and their impact factors. Journal of Documentation, 54(4), 387-419.

Moed, H. F., \& Visser, M. S. (2007). Developing bibliometric indicators of research performance in computer science: An exploratory study. Research Report to the Council for Physical Sciences of the Netherlands Organisation for Scientific Research. Hague: Netherlands Organisation for Scientific Research.

Murugesan, P., \& Moravcsik, M. J. (1978). Variation of nature of citation measures with journals and scientific specialties. Journal of the American Society for Information Science, 29(3), 141-147.

Nederhof, A. J. (2005). Bibliometric monitoring of research performance in the social sciences and the humanities: A review. Scientometrics, 66(1), 81-100. doi:10.1007/s11192-006-0007-2.

Opthof, T., \& Leydesdorff, L. (2010). Caveats for the journal and field normalizations in the cwts ("leiden") evaluations of research performance. Journal of Informetrics, 4(3), 423-430.

Pinski, G., \& Narin, F. (1976). Citation influence for journal aggregates of scientific publications-theory, with application to literature of physics. Information Processing \& Management, 12(5), 297-312.

Shepherd, P. T. (2010). The feasibility of developing and implementing journal usage factors: A research project sponsored by UKSG. The Journal of Serials Community, 20, 117-123.

Small, H., \& Sweeney, E. (1985). Clustering the science citation index using co-citations. 1. A comparison of methods. Scientometrics, 7(3-6), 391-409.

Sombatsompop, N., Markpin, T., Yochai, W., \& Saechiew, M. (2005). An evaluation of research performance for different subject categories using impact factor point average (IFPA) index: Thailand case study. Scientometrics, 65(3), 293-305. doi:10.1007/s11192-005-0275-2.

Van Leeuwen, T. N., \& Moed, H. F. (2002). Development and application of journal impact measures in the Dutch science system. Scientometrics, 53(2), 249-266.

Vieira, E. S., \& Gomes, J. A. N. F. (2010). Citations to scientific articles: Its distribution and dependence on the article features. Journal of Informetrics, 4(1), 1-13. doi:10.1016/j.joi.2009.06.002.

Zitt, M., \& Small, H. (2008). Modifying the journal impact factor by fractional citation weighting: The audience factor. Journal of the American Society for Information Science and Technology, 59(11), 1856-1860. doi:10.1002/asi.20880. 